\title{
Order-disorder transitions at and segregation to (001) Ni-Pt surfaces
}

\author{
R. Najafabadi and D.J. Srolovitz \\ Department of Materials Science \& Engineering, University of Michigan, Ann Arbor, MI 48109, USA
}

Received 8 October 1992; accepted for publication 22 December 1992

\begin{abstract}
Order-disorder transitions at and segregation to the (001) surface of $\mathrm{Ni}-\mathrm{Pt}$ alloys have been investigated by a recently developed free energy simulation method, where the atomic interactions are described using the embedded atom method (EAM) potentials. On the Ni-rich side of the phase diagram, we observe a second order, order-disorder phase transition on the (001) surfaces at temperatures well above the bulk phase transition temperature. At the transition temperature, the first (002) atomic plane changes from a disordered plane to an ordered one with the $c(2 \times 2)$ pattern. The second $(002)$ plane changes from a disordered plane to a nearly pure Ni plane. Subsequent planes retain their essentially bulk-like, disordered structure. We also observe first order, order-disorder surface phase transitions on the Pt-rich side of the phase diagram. At the transition temperatures, the first and third (002) planes become nearly pure $\mathrm{Pt}$ and the second plane becomes nearly pure Ni. The effect of the surface transitions on the thermodynamic properties of the surfaces, such as enthalpy and vibrational and configurational entropy, are also investigated. It is shown that the ordering surface transition upon cooling the Ni-rich sample is enthalpically driven. The present simulations also show the importance of including atomic vibrations in surface segregation studies. Atomic vibrations have been typically omitted in previous lattice gas descriptions of surface segregation.
\end{abstract}

\section{Introduction}

Structure and composition of surfaces in alloys are of great importance in catalysis, corrosion, etc. The properties and behavior of surfaces are sensitive to variations in the properties of the bulk material. Nonetheless, the structure and composition of surfaces can be very different from those in the bulk due to the different atomic environment at the surface. The change in atomic environment may lead to surface relaxation, reconstruction, segregation, and phase transitions. For example, the (001) surfaces in $\mathrm{Au}$-rich $\mathrm{Cu}_{3} \mathrm{Au}$ [1] and stoichiometric $\mathrm{Cu}_{3} \mathrm{Au}$ [2] alloys have been observed to remain ordered at temperatures above the bulk order-disorder transition temperature, where the bulk alloy is disordered. Surface phase transitions associated with surface miscibility gaps have been observed at the (111) surfaces of $\mathrm{Ag}-\mathrm{Cu}$ [3,4] alloys. Several calculations [5-11] have been performed to investigate surface segregation in binary systems. Many of these studies were based on the lattice gas model, which as- sumes fixed bond strengths, all atoms at perfect crystal locations and ignores the vibrational effects on the surface segregation. In these studies the surface relaxation is either ignored or is included simply as a variational parameter. In this study, we employ a recently developed [12] free energy simulation method and the embedded atom method (EAM) interatomic potentials [13] to study the behavior of (001) surfaces in two $\mathrm{Ni}-\mathrm{Pt}$ alloys. Within the framework of the free energy simulation method, the atomic vibration, atomic relaxation, and atomic site occupancies are all consistently determined from the EAM description for the atomic interactions.

Surface segregation in the $\mathrm{Ni}-\mathrm{Pt}$ binary alloys has recently received extensive experimental attention [14-18]. The (001) and (111) surfaces of the alloy with equal $\mathrm{Ni}$ and Pt content was experimentally found to be strongly enriched in platinum, while the next atomic plane under the surface is enriched in nickel $[14,16]$. Deeper atomic planes were found to have essentially the bulk composition. On the other hand, the same 
experimental techniques have shown the reverse segregation tendencies at the (110) surface; the first plane becomes strongly enriched in nickel while the second plane is enriched in platinum. Similar surface segregation behavior has been observed in the $10 \%$ platinum alloys. All of these measurements were made on samples which were annealed at temperatures well above the critical temperature for this alloy $(918 \mathrm{~K})$. There have been several theoretical studies of surface segregation in the $\mathrm{Ni}-\mathrm{Pt}$ binary system $[5,9]$ which are in reasonable agreement with the experiments. However, in many cases these simulations were not performed in the same region of the phase diagram as the experiments. In some of these studies [9], the simulations were performed within the two phase region of the phase diagram (for the interatomic potentials employed) with the bulk artificially constrained to be disordered. It were these results which were compared with the experimental data corresponding to equilibrium at temperatures much higher than the bulk order-disorder temperature.

In the present paper we examine the orderdisorder phase transitions at and segregation to (001) surfaces in the $\mathrm{Ni}-\mathrm{Pt}$ alloy system using EAM potential for Ni-Pt binary alloys. Care was taken to guarantee that the simulations were all performed within the correct region of the phase diagram for these interatomic potentials. A series of simulations were performed in both $\mathrm{Ni}$ and Pt-rich alloys at a series of temperature from much above $T_{\mathrm{x}}$ to very close to the two phase region. In the following section, we describe the free energy simulation method and present the equilibrium phase diagram for the EAM Ni-Pt alloy system. The effects of atomic vibration and surface relaxation on the surface segregation are presented in section 3. We show, in section 4, how the (001) surface segregation changes as the bulk phase transition temperatures are approached.

\section{Method}

The local harmonic (LH) model [19] has been applied with considerable success to perfect and defected single component solids [20,21]. In this model, the classical vibrational contribution to the free energy for a single component system is given by

$$
A_{\mathrm{v}}=k_{\mathrm{B}} T \sum_{i=1}^{N} \sum_{\beta=1}^{3} \ln \left(\frac{h \omega_{i \beta}}{2 \pi k_{\mathrm{B}} T}\right),
$$

where $k_{\mathrm{B}} T$ is the thermal energy, $h$ is Planck's constant, $N$ is the total number of atoms in the system, and $\omega_{i 1}, \omega_{i 2}$, and $\omega_{i 3}$ are the three vibrational frequencies of atom $i$. These frequencies may be determined in terms of the local dynamical matrix of each atom $D_{i \alpha \beta}=\left(\partial^{2} E / \partial x_{i \alpha} \partial x_{i \beta}\right)$, where the $x_{i \beta}$ correspond to displacements of atom $i$ in some coordinate system.

In order to study binary alloys within the frame work of the $\mathrm{LH}$ model, an effective atom is assigned to each atomic site which has an effective mass $m_{i}=x_{\mathrm{a}}(i) m_{\mathrm{a}}+x_{\mathrm{b}}(i) m_{\mathrm{b}}$. Here, $x_{\mathrm{a}}(i)$ is the probability that atomic site $i$ is occupied by an atom of type "a" and, correspondingly, $x_{\mathrm{b}}(i)=1$ $-x_{\mathrm{a}}(i)$ is the probability that the same atomic sitc is occupicd by an atom of typc " $b$ ". The vibrational contribution to the free energy is determined from the appropriately averaged local dynamical matrix (for details see ref. [12]). The configurational entropy is written using the Bragg-Williams or point approximation as

$$
\begin{aligned}
S_{\mathrm{c}}= & -k_{\mathrm{B}} \sum_{i=1}^{N}\left\{x_{\mathrm{a}}(i) \ln \left[x_{\mathrm{a}}(i)\right]\right. \\
& \left.+x_{\mathrm{b}}(i) \ln \left[x_{\mathrm{b}}(i)\right]\right\} .
\end{aligned}
$$

In the simulations described below, we employ a reduced grand canonical ensemble, where the total number of atoms remains fixed but the relative quantities of each atomic species varies. The appropriate thermodynamic potential for this type of ensemble is the grand potential and is given by

$$
\begin{aligned}
\Omega & =A+\Delta \mu \sum_{i=1}^{N} x_{\mathrm{a}}(i) \\
& =E+A_{\mathrm{v}}-T S_{\mathrm{c}}+\Delta \mu \sum_{i=1}^{N} x_{\mathrm{a}}(i),
\end{aligned}
$$


where $A$ is the Helmholtz free energy, $E$ is the static energy (obtained via an interatomic potential), and $\Delta \mu$ is the difference in chemical potential between atoms of type "a" and "b". Given $\Delta \mu$, the equilibrium concentration at each site can be determined by minimizing $\Omega$ with respect to the site concentrations $x_{\mathrm{a}}(i)$.

Surface free energy in this ensemble is defined by the following equation:

$$
\gamma_{\mathrm{s}}(\Delta \mu, T)=\left[\Omega_{\mathrm{s}}(\Delta \mu, T)-\Omega_{\mathrm{p}}(\Delta \mu, T)\right] / A_{\mathrm{s}}
$$

where $\Omega_{\mathrm{s}}$ and $\Omega_{\mathrm{p}}$ are grand potential energies of the surface and perfect system, respectively. The surface free energy is normalized by the surface area, $A_{\mathrm{s}}$.

The geometry of the simulation cell used in this study is described in detail elsewhere [19]. Parallel to the surface, periodic boundary conditions are employed. The simulation cell in the plane of the surface was $4 a_{0} \times 4 a_{0}$, where $a_{0}$ is the equilibrium lattice parameter. The simulation cell is bonded to an infinite block of structurally perfect crystal far from the surface. In the direction perpendicular to the surface, at least 10 (002) planes were allowed to relax. The lattice constant and site concentrations in this block are held fixed during the course of simulation and have the same values as those determined at the desired temperature and chemical potential difference for the bulk phase.

In the present simulations, the atomic interactions were modeled using the well known EAM alloy potentials (see ref. [13] for details). These potentials were fit to bulk thermodynamic, vacancy formation energy, and dilute heat of solution data. The solid portion of the calculated phase diagram for the $\mathrm{Ni}-\mathrm{Pt}$ alloy system determined using the EAM interatomic potentials and the free energy simulation, described above, is shown in fig. 1. We note that the phase diagram for the $\mathrm{Ni}-\mathrm{Pt}$ model system is not in quantitative agreement with the experimental phase diagram. This is partly due to the assumptions made in the approximation of the free energy and errors in the EAM description of the atomic interactions. The predicted structure for the $\mathrm{Ni}_{3} \mathrm{Pt}$ phase is

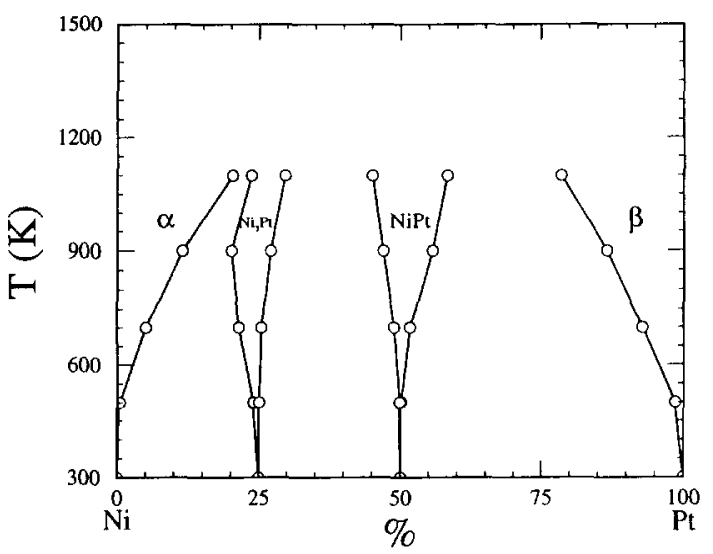

Fig. 1. The solid regions of the bulk Ni-Pt phase diagram determined using the free energy simulation method employing EAM potentials.

$\mathrm{L1}_{2}\left(\mathrm{Cu}_{3} \mathrm{Au}\right)$, in agreement with experiment. The model correctly predicts the absence of the $\mathrm{Pt}_{3} \mathrm{Ni}$ phase. However, the model predicts that the stable $\mathrm{Ni}-\mathrm{Pt}$ phase is $\mathrm{B} 2(\mathrm{CsCl})$ rather than the experimentally observed $\mathrm{L1}_{0}$ (CuAu) structure. In this study, we are interested in understanding the surface segregation and surface phase transition behavior in a binary alloy as one approaches the two phase regions on both the Ni-rich and the Pt-rich sides of the model phase diagram from high temperature. Therefore, while the results for the $\mathrm{Ni}$-rich side of the $\mathrm{Ni}$-Pt phase diagram should be reliable, the data obtained on the Ptrich side of the diagram should be viewed more as a model study and only compared with experiment with due caution.

\section{Atomic vibration and surface relaxation effects on segregation}

Before we consider the variation in the surface segregation with temperature specially as one approaches the bulk two phase regions, we first examine the effect of atomic vibration and surface relaxation on surface segregation. As mentioned above, most previous theoretical studies of surfaces segregation in alloys neglected both atomic vibrations at finite temperature and/or surface relaxation. However, both of these effects 
Table 1

Pt atomic site concentrations on the top six (002) planes $x_{n}$ and changes in the interplanar spacing $\Delta_{n, n+1}$ for a Ni-Pt alloy with $10 \% \mathrm{Pt}$ in the bulk at $1500 \mathrm{~K}$

\begin{tabular}{|c|c|c|c|c|c|c|c|c|c|}
\hline & \multicolumn{6}{|c|}{$\mathrm{Pt}$ atomic site concentration $(\% \mathrm{Pt})$} & \multicolumn{3}{|c|}{ Changes in interplanar spacing (\%) } \\
\hline & $x_{1}$ & $x_{2}$ & $x_{3}$ & $x_{4}$ & $x_{5}$ & $x_{6}$ & $\Delta_{12}$ & $\Delta_{23}$ & $\Delta_{34}$ \\
\hline Case A: unrelaxed & 24.05 & 7.01 & 10.97 & 9.72 & 10.08 & $9 . \overline{98}$ & & & \\
\hline Case B: positions & 23.61 & 5.23 & 11.14 & 10.59 & 10.16 & 10.09 & -0.74 & -0.30 & +0.16 \\
\hline Case C: vibrations & 11.97 & 9.58 & 10.09 & 9.98 & 10.00 & 10.00 & & & \\
\hline Case D: fully relaxed & 11.94 & 9.35 & 10.17 & 10.00 & 10.02 & 10.01 & -0.22 & 0.00 & +0.03 \\
\hline
\end{tabular}

The data labeled case $A$ is determined with all of the atoms frozen at their $T-0 \mathrm{~K}$ atomic positions and atomic vibrations are ignored. The data in case $B$ was determined by minimizing the free energy with respect to atomic positions and site concentrations, however, the vibrational contribution to the free energy was omitted from the free energy. Case $\mathrm{C}$ is identical with case $\mathrm{A}$, except that atomic vibrations are included in the free energy expression. The data in case $D$ was determined by relaxing the free energy (including the atomic vibration contribution) with respect to atomic positions and site concentrations.

are fully accounted for in the Monte Carlo studies (see, for example the $\mathrm{Cu}-\mathrm{Ni}$ alloy surface segregation study in ref. [6]) in which all of the atoms are free to move in throughout all of phase space. We have previously demonstrated [12] that the segregation profiles determined for several different surface orientations in $\mathrm{Cu}-\mathrm{Ni}$ using the approximate free energy simulation method are in very good agreement with the more accurate Monte Carlo results using the same interatomic potentials.

In order to determine the effect of atomic vibration and surface relaxation on the (001) surface segregation profile, we have performed a series of calculations at bulk compositions of $x_{\mathrm{B}}$ $=10 \%$ platinum at $1500 \mathrm{~K}$ employing different constraints: case $\mathbf{A}$ - no atomic relaxations and no atomic vibrations, case $B$ - with atomic relaxation but without atomic vibrations, case $\mathrm{C}$ - with atomic vibrations but no atomic relaxations, and case $\mathrm{D}$ - with atomic relaxation and atomic vibrations. The composition profile is determined by minimizing the grand potential, with respect to the site concentrations for cases $\mathrm{A}$ and $\mathrm{C}$, and with respect to the site concentrations and atom positions for cases $\mathrm{B}$ and $\mathrm{D}$. If atomic vibrations are not included, the vibrational term in the grand potential $A_{\mathrm{v}}$ is set to zero. Table 1 shows the Pt concentration (at \%) on the top six (002) planes and the change in the interplanar spacings $\Delta_{n, n+1}$ (between the $n$th and $n+1$ st atomic planes) that occurs upon relaxation. Comparison of the results from cases $A$ and $B$ show that atomic relaxations do not significantly change the segregation profile. This is particularly important since the difference in the segregation to the first layer $\left(x_{1}-x_{\mathrm{B}}\right)$ between the unrelaxed and fully relaxed cases is of order $700 \%$ (cf. cases A, B and D). Similarly, we find that the changes in the interplanar spacings when the atomic positions are relaxed but the atomic vibrations are not included are also in poor agreement with the fully relaxed (position and vibrations) surface (cf. cases $\mathrm{B}$ and D). Comparison of cases $\mathrm{A}$ and $\mathrm{C}$ demonstrate that inclusion of the vibrational entropy has a profound effect on the equilibrium segrega-

Table 2

As in table 1, but for the (111) surface of Ni-Pt where the bulk Pt concentration was $10 \%$ and $T=1500 \mathrm{~K}$

\begin{tabular}{|c|c|c|c|c|c|c|c|c|c|}
\hline & \multicolumn{6}{|c|}{$\mathrm{Pt}$ atomic site concentration $(\% \mathrm{Pt})$} & \multicolumn{3}{|c|}{ Changes in interplanar spacing (\%) } \\
\hline & $\overline{x_{1}}$ & $x_{2}$ & $x_{3}$ & $x_{4}$ & $x_{5}$ & $x_{6}$ & $\Delta_{12}$ & $\Delta_{23}$ & $\Delta_{34}$ \\
\hline$\overline{\text { Case } A \text { : unrelaxed }}$ & 36.23 & 2.44 & 14.46 & 7.97 & 10.96 & 9.59 & & & \\
\hline Case B: positions & 35.30 & 1.06 & 11.33 & 10.11 & 9.97 & 10.00 & -1.11 & -1.00 & +0.16 \\
\hline Case C: vibrations & 12.62 & 8.69 & 10.53 & 9.93 & 10.05 & 9.99 & & & \\
\hline Case D: fully relaxed & 12.71 & 8.39 & 10.44 & 9.92 & 10.01 & 10.00 & -0.12 & -0.16 & +0.03 \\
\hline
\end{tabular}


tion profile. Furthermore, the excellent agreement between the segregation profiles for cases $\mathrm{C}$ (with atomic vibrations) and D (fully relaxed), further demonstrates that inclusion of the vibrational entropy is the key and that surface relaxation has little effect and could be ignored in determining surface segregation profiles. These results further suggest that ignoring atomic vibrations can lead to very large errors in determining the degree of segregation.

We have also carried out a set of similar calculations for (111) surfaces in the Ni-Pt alloy system at the same temperature and bulk composition as for the (001) surface, reported above. The results for the segregation profiles and the change in the interplanar spacing are summarized in table 2. Again, comparison of the different sets of data clearly show that the effects of surface relaxation on the segregation profiles is negligible and inclusion of the atomic vibrations is crucial in obtaining reasonable segregation profiles. In fact, the effects of atomic vibrations is even more pronounced on the (111) than it is on the (001) surfaces.

\section{4. (001) surface thermodynamics and composition}

\subsection{Ni-rich alloys}

The ordered alloy with the highest $\mathrm{Ni}$ concentration is $\mathrm{Ni}_{3} \mathrm{Pt}$ (see fig. 1), which has the $\mathrm{Ll}_{2}$ or $\mathrm{Cu}_{3} \mathrm{Au}$ structure. This structure is isomorphic with the face centered cubic lattice, where $\mathrm{Pt}$ atoms occupy the corners of the cubic unit cell and $\mathrm{Ni}$ occupies the faces. Alternatively, this structure may be described as consisting of four interpenetrating simple cubic lattices. We find that the stable (001) surface at low temperatures consists of two interpenetrating square lattices $c(2 \times 2)$; one is Ni-rich while the other is Pt-rich.

At the highest temperature studied, $T=1500$ $\mathrm{K}$, the segregation profile for the $(001)$ surface in the $x_{\mathrm{B}}=5 \%$ Pt bulk composition alloy shows that the first $(002)$ plane is enriched in platinum with an average composition of $8.1 \% \mathrm{Pt}$ (see fig. $2 \mathrm{a}$ ). The Pt concentration on the second $(002)$ plane is
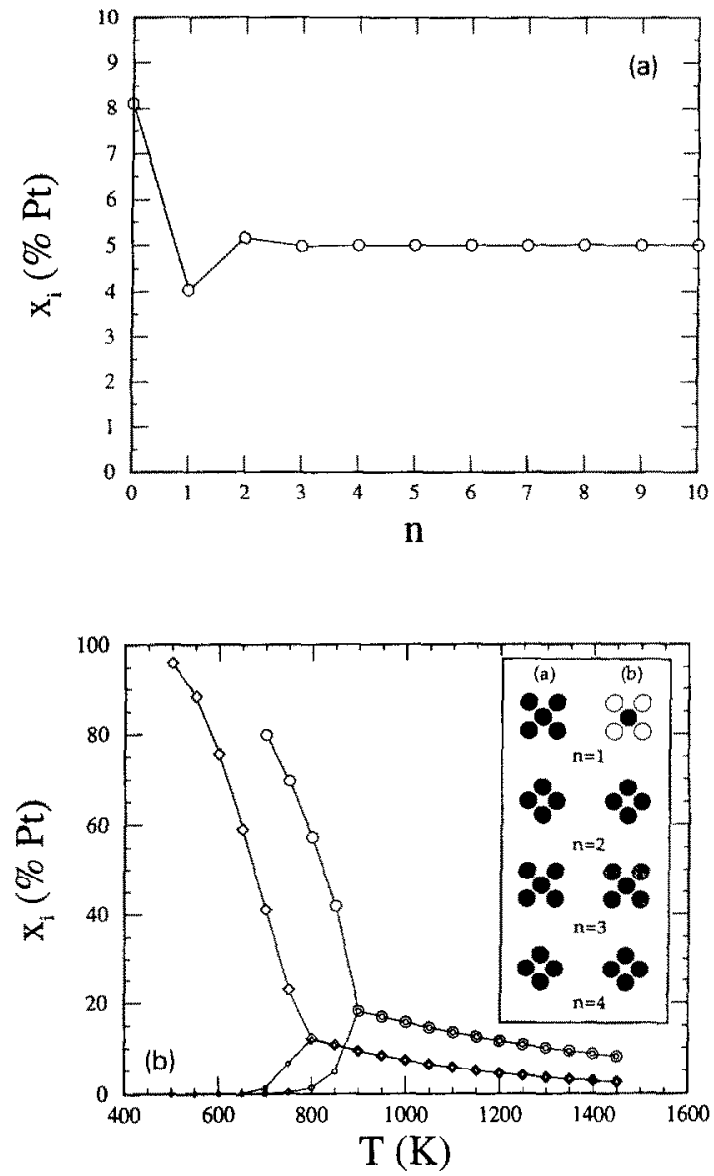

Fig. 2. (a) The variation of the average $(002)$ atomic plane $P t$ concentration as a function of plane number (distance from the surface) for $5 \% \mathrm{Pt}$ and $T=1500 \mathrm{~K}$. (b) The average sublattice concentrations on the first $(002)$ plane as a function of temperature for $x_{\mathrm{B}}=5 \%$ (circles) and $x_{\mathrm{B}}=1 \% \mathrm{Pt}$ (diamonds). The large and small symbols represent concentrations on two different sublattices of the (001) surface plane. The inset shows the top four (002) planes of the disordered (a) and ordered (b) surface phase. The grayness represents the average site concentration which may vary from black to white corresponding to pure $\mathrm{Ni}$ and $\mathrm{Pt}$, respectively.

slightly enriched in nickel with an average $\mathrm{Pt}$ concentration of $4 \%$. More deeply buried (002) planes have essentially the same composition as the bulk $(5 \% \mathrm{Pt})$. As the temperature is decreased from 1500 to $900 \mathrm{~K}$ in 50 uniform temperature increments at fixed bulk composition in the 5\% Pt alloy, the segregation to the first $(002)$ 
plane increases to approximately $20 \%$ and all of the sites on the surface have the same composition: the (001) surface is compositionally disordered in this temperature range. At $900 \mathrm{~K}$, the second (002) plane concentration is approximately $1 \% \mathrm{Pt}$ and the third (002) plane is about $6 \% \mathrm{Pt}$ and the subsequent planes are at the bulk composition. In general, as the temperature is lowered from $1500 \mathrm{~K}$ to $900 \mathrm{~K}$, the $\mathrm{Pt}$ concentration on the first (002) plane and the $\mathrm{Ni}$ concentration on the second (002) plane increase (see fig. $2 b$ ).

When the temperature is decreased to below $900 \mathrm{~K}$, we observe two distinct site concentrations on the surface. 'These two types of atomic sites are arranged in a $\mathrm{c}(2 \times 2)$ (ordered mixed layer) structure in which the corncrs of the squarcs are all alike and are Ni-rich, while the center atomic sites are indistinguishable and are Pt-rich. Therefore, the surface undergoes a phase transition from the disordered $(1 \times 1)$ structure to the ordered $c(2 \times 2)$ structure as the (001) surface is cooled through $900 \mathrm{~K}$. At $800 \mathrm{~K}$, the Pt concentrations on the two sublattices of the first $(002)$ plane are about $1 \%$ and $58 \%$. Subsequent decrease in temperature to $700 \mathrm{~K}$ (the calculated bulk phase transition temperature for $5 \% \mathrm{Pt}$ is approximately $700 \mathrm{~K}$ - see fig. 1) does not qualitatively change the segregation profile. At $850 \mathrm{~K}$ and below, the second (002) atomic plane exhibits nearly uniform concentration, somewhat enriched in Ni. By the third (002) plane, the Pt concentration approaches its bulk value - however, it exhibits inequivalent site concentrations in the same pattern as the surface. Beyond the third (002) plane, the site concentrations are essentially at their bulk values and no in-plane variations are observed. The concentration distribution within the first three (002) planes at $900 \mathrm{~K} \geq T \geq 700 \mathrm{~K}$ is reminiscent of the $\mathrm{L}_{2}$ ordering in the bulk below its order-disorder temperature $(\sim 700 \mathrm{~K})$.

Fig. $2 b$ also shows the results of simulations performed at a bulk concentration of $1 \% \mathrm{Pt}$. At this bulk composition, we find a similar (002) surface phase transition from the disordered high temperature phase to a $\mathrm{c}(2 \times 2)$ ordered surface at low temperature. This surface phase transition occurs at $T \approx 800 \mathrm{~K}$, as compared with the bulk

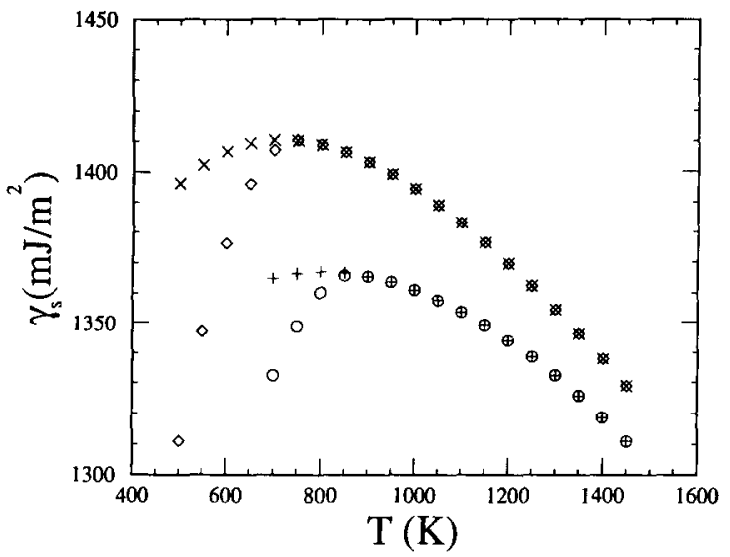

Fig. 3. Surface free energy as a function of temperature for $x_{\mathrm{B}}=5 \%$ Pt $((O)$ and $(+))$ and $x_{\mathrm{B}}=1 \%$ Pt $((\diamond$ and $(x))$. The $(+)$ and $(X)$ correspond to data obtained on cooling, while the $(0)$ and $(\diamond)$ correspond to heating.

ordering temperature of approximately $525 \mathrm{~K}$ in the $1 \%$ alloy.

In order to determine the surface phase transition temperatures more accurately and to identify the order of the surface phase transitions we have calculated the surface free energies of the two competing surface phases (when they are either stable or metastable). We note that the disordered phase observed at low temperatures is the undercooled high temperature surface phase and it remains stable against infinitesimal spatial concentration fluctuation, i.e. it is truly metastable. In fig. 3 , we show the variation in the surface free energies for the ordered and disordered surfaces with decreasing and increasing temperature for both $5 \%$ and $1 \% \mathrm{Pt}$ bulk compositions. Based on figs. 2 and 3 surface phase transition temperatures are determined to be about 875 and $775 \mathrm{~K}$ for the 5\% and 1\% Pt bulk composition, respectively. Since the slopes of the surface free energy curves for the two competing surface phases are indistinguishable at the transition temperatures, we conclude that this order-disorder surface phase transition is second order. On cooling through the surface transition temperature, we find that it is possible to obtain a metastable disordered phase. However, heating through the transition temperature, we find that the low temperature ordered phase is not even metastable. The lack of a metastable phase on both sides of 
the transition temperalure indicates that this is not a first order transition and the presence of a metastable phase on one side of the transition temperature is not inconsistent with a second order transition.

\subsection{Pt-rich alloys}

As discussed in section 2, the EAM Ni-Pt alloy potentials do not yield the experimentally observed NiPt structure. While use of the EAM interatomic potentials predicts that the $\mathrm{B} 2(\mathrm{CsCl})$ structure is stable, the experimentally observed structure $\mathrm{NiPt}$ is $\mathrm{L1}_{0}$ (alternating $\mathrm{Ni}$ and $\mathrm{Pt}(002)$ layers in a nominally fcc lattice). Our simulations on $\mathrm{Ni}$-rich $\mathrm{Ni}-\mathrm{Pt}$ alloys indicated that the observed surfacc phases that occur close to, but above, the two phase region are intimately related to the phases present in the lower temperature two phase region. Therefore, the applicability of the simulation results on the Pt-rich side of the phase diagram must be viewed with due caution. Nonetheless, we note that in some closely related alloy systems, e.g., in CuPd, the B2 is the stable structure. As a result, we believe that the results obtained on the Pt-rich side of the $\mathrm{Ni}-\mathrm{Pt}$ phase diagram should be viewed as prototypical of a different class of order-disorder transitions than we observed on the Ni-rich end of the phase diagram, where both the ordered and disordered phase share the same fundamental lattice type fcc. This other class of order-disorder surface phase transitions is one from a disordered high temperature phase ( $f(c)$ to an ordered low temperature phase with a different fundamental lattice type (i.e., the B2-CsCl structure shares the same fundamental lattice type with a bcc crystal).

Fig. 4 shows the variation of the platinum concentration on the first and second (002) planes with temperature at $x_{\mathrm{B}}=95 \% \mathrm{Pt}$ and $x_{\mathrm{B}}=99 \%$ $\mathrm{Pt}$, as the temperature is reduced from the high temperature disordered phase towards the two phase region of the phase diagram (see fig. 1). The concentrations within each (002) plane are uniform. For the $x_{\mathrm{B}}=95 \% \mathrm{Pt}$ alloy at $1500 \mathrm{~K}$, we observe the nickel concentration on the first and second (002) planes increases by 3 and $5.5 \%$, respectively, while it is decreased on the third

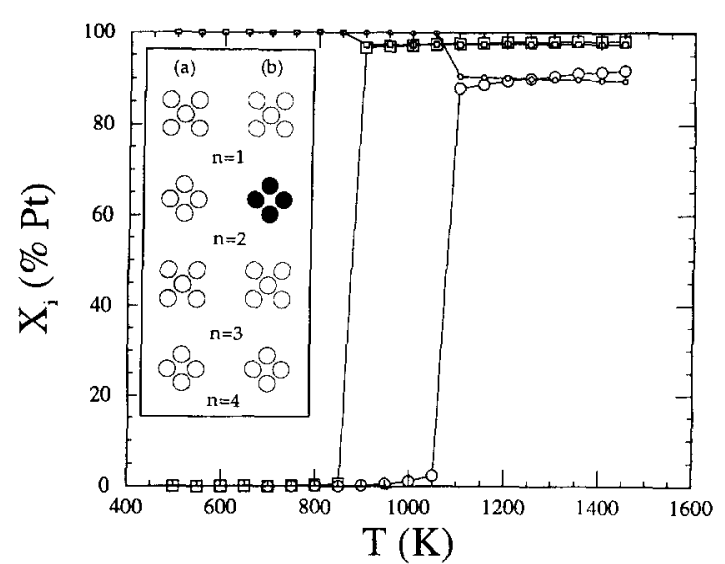

Fig. 4. The average concentrations on the first two (002) plane as a function of temperature for $x_{B}=95 \%$ (circles) and $x_{\mathrm{B}}=99 \% \mathrm{Pt}$ (diamonds). The large and small symbols represent concentrations on the first and second (002) plane, respectively. The inset shows the top four $(002)$ planes of the disordered (a) and ordered (b) surface phase. The grayness represents the averaged site concentration which may vary from black to white corresponding to pure $\mathrm{Ni}$ and $\mathrm{Pt}$, respectively.

(002) plane by $0.5 \%$. The compositions of subsequent (002) planes are essentially the same as in the bulk. As we decrease the temperature from 1500 to $1050 \mathrm{~K}$ in 50 increments, the segregation profile remains essentially unchanged except that the nickel concentration on the second (002) plane increases and becomes larger than that on the first (002) plane at $\sim 1200 \mathrm{~K}$.

With further decreases in the temperature below $1050 \mathrm{~K}$, we observe that the Pt concentration on the second (002) plane drops from nearly $83 \%$ at $1050 \mathrm{~K}$ to only $0.3 \%$ at $1000 \mathrm{~K}$. When the $\mathrm{Pt}$ concentration on the second (002) drops, the first and third (002) planes become nearly pure platinum and the fourth (002) plane becomes slightly enriched in platinum with no significant changes in the concentration of the subsequent planes. Subsequent decreases in the temperature down to $650 \mathrm{~K}$ do not result in significant further changes in this segregation profile. Below $1050 \mathrm{~K}$, the concentration profile on the first three $(002)$ atomic planes that we observe are compatible with the concentrations on the $(002)$ planes of the equilibrium $\mathrm{B} 2$ and with the $\mathrm{L}_{0}$ structure. Ac- 


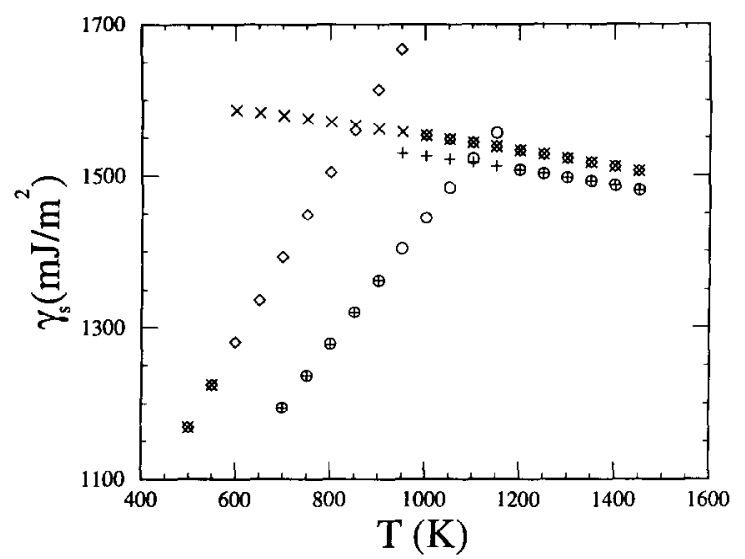

Fig. 5. Surface free energy as a function of temperature for $x_{\mathrm{B}}=95 \% \mathrm{Pt}((\circ)$ and $(+))$ and $x_{\mathrm{B}}=99 \% \mathrm{Pt}((\diamond)$ and $(x))$. The $(+)$ and $(x)$ correspond to data obtained on cooling, while the $(0)$ and $(\diamond)$ correspond to heating.

companying this phase transition from the disordered to ordered surfaces in the Pt-rich alloys, we also observe a discontinuous change in the spacing between the first and second (002) atomic planes and between the second and third (002) planes.

We have performed a series of similar calculations in the $x_{\mathrm{B}}=99 \% \mathrm{Pt}$ alloy. The behavior observed in this alloy is essentially the same as in the $x_{\mathrm{B}}=95 \% \mathrm{Pt}$ alloy (see fig. 4 ) and the orderdisorder transition occurs near $850 \mathrm{~K}$.

The variation of the surface free energy with temperature is shown in fig. 5 for bulk compositions of $95 \%$ and $99 \%$ Pt. Two sets of calculations were performed: by heating the system from well below the equilibrium transition temperature (where the surface is ordered) and by cooling the sample through the surface order-disorder temperature starting at very high temperature (where the surface is disordered). The surface transition temperatures, defined as the points where the surface free energy curves for the two competing surfaces cross, are 1100 and $850 \mathrm{~K}$ for bulk concentrations of $95 \%$ and $99 \% \mathrm{Pt}$, respectively. The discontinuity in the slopes of the free energy curves at the order-disorder temperature indicates that this is a first order phase transition at both bulk compositions examined. This is consistent with the observed variation in the $(002)$ plane concentrations (fig. 4) with temperature - a discontinuous change in the order parameter (site occupancies) is indicative of a first order phase transition. On cooling the $95 \% \mathrm{Pt}(99 \% \mathrm{Pt})$ system from the disordered state, we find that the surface does not become ordered until $\sim 900 \mathrm{~K}$ $(\sim 675 \mathrm{~K})$. On heating the $95 \% \mathrm{Pt}(99 \% \mathrm{Pt})$ system from the ordered state, we find that the surface does not become disordered until $\sim 1175$ $\mathrm{K}(\sim 975 \mathrm{~K})$. This hysteresis on temperature cycling is another clear indication of a first order surface phase transition.

\section{Discussion and conclusions}

The surface phase transitions obscrved in the $\mathrm{Ni}-\mathrm{Pt}$ binary model system are intimately related to the phases present in the two phase regions for the same bulk composition. For the ordered surface phase on the Ni-rich side of the phase diagram, the concentrations of the two sublattices on the first (002) plane are 69.70 and $0.35 \% \mathrm{Pt}$ when the bulk composition is $5 \% \mathrm{Pt}$ at $750 \mathrm{~K}$. All of the atomic sites on the second (002) plane have an average composition of $0.1 \% \mathrm{Pt}$, while the average compositions on the two sublattices present on the third (002) plane are 6.4 and $5.5 \% \mathrm{Pt}$. The (001) surface ripples, with the Ni-rich sites being displaced towards the bulk by approximately 0.031 $\AA$ more than the Pt-rich sites at $750 \mathrm{~K}$. The ordering and concentrations on the first two (002) planes are similar to the two different (002) planes in the $\mathrm{Ni}_{3} \mathrm{Pt} \mathrm{L1}_{2}$ ordered phase (see inset in fig. $2 \mathrm{~b})$. Although the composition of the two sublattices represented on the third (002) plane are very close, they are not identical and are arranged such that the Pt-rich site on the third (002) plane is consistent with the $\mathrm{L1}_{2}$ ordering observed on the first and second (002) planes. The surface ordering is essentially limited to the first two (002) planes and does not grow until the two phase region of the bulk phase diagram is reached. In order to examine the stability of the width of this ordered surface region, we performed a series of simulations at $750 \mathrm{~K}$ and $x_{\mathrm{B}}=5 \% \mathrm{Pt}$ in which we started with different numbers of ordered (002) planes (consistent with 

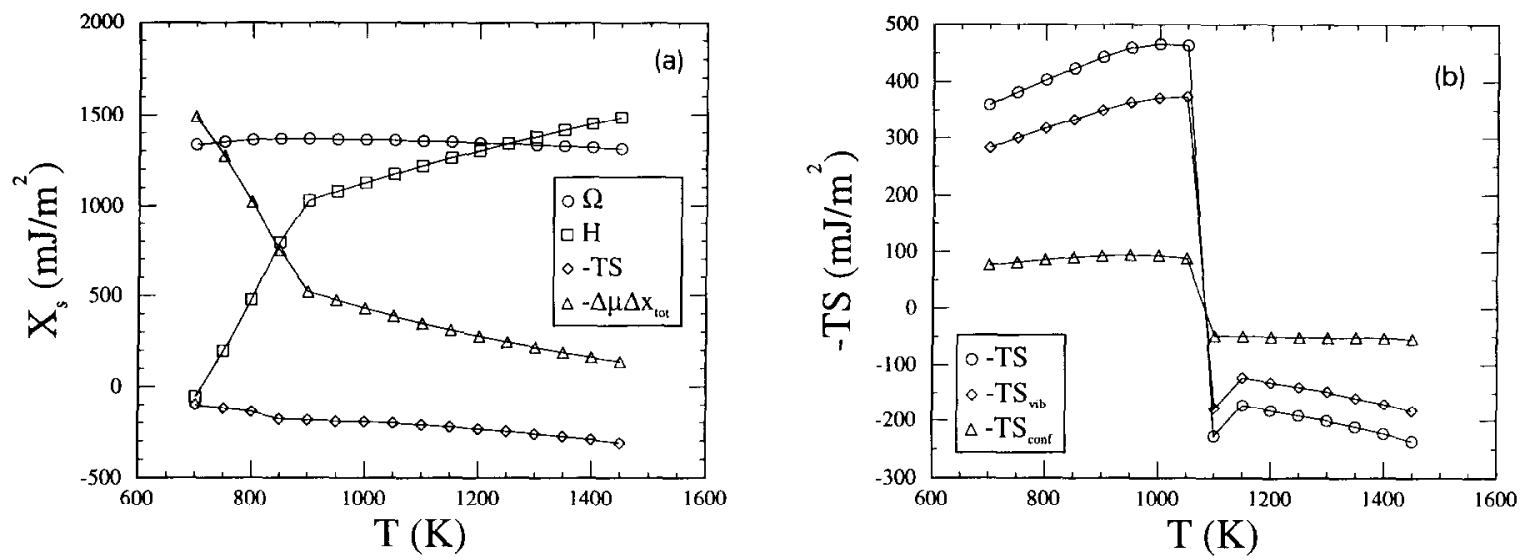

Fig. 6. The surface free energy and its enthalpic $H$, entropic $-T S$ and segregation components $-\Delta \mu \Delta x_{\text {tor }}$ as a function of temperature for (a) $x_{\mathrm{B}}=5 \% \mathrm{Pt}$ and (b) $x_{\mathrm{B}}=95 \% \mathrm{Pt}$.

the $\mathrm{L1}_{2}$ ordering) at the surface. We found that when these structures were relaxed, the resultant concentration distributions were the same and were consistent with those reported above. There is some experimental evidence [16] which shows partial compositional ordering on the (001) surface with $x_{\mathrm{B}}-10 \% \mathrm{Pt}$ which is similar to the surface ordering reported above.

The ordered (001) surfaces observed in the Pt-rich side of the phase diagram are also closely related to the corresponding two phase region. As discussed above, the EAM potentials for $\mathrm{Ni}-\mathrm{Pt}$ alloys do not predict the correct structure for the $\mathrm{NiPt}$ phase. At $750 \mathrm{~K}$ and $x_{\mathrm{B}}=95 \% \mathrm{Pt}$, the concentrations within each (002) plane near the ordered surface are uniform and the first four (002) plane compositions are 100.00, 0.01, 99.96, and $96.13 \% \mathrm{Pt}$. The concentrations on the first two (002) planes of the ordered surface are similar to those observed in the equilibrium NiPt (B2) ordered phase (see inset in fig. 4). When the ordering occurs, the interplanar spacing between the top two (002) planes decreases significantly ( $\sim 13 \%$ of the bulk fcc lattice constant). This pronounced decrease in the interplanar spacing can be attributed to the difference in the lattice constants of the NiPt alloy in the ordered (B2) and disordered $\mathrm{fcc}$ phase. The [100] and [010]
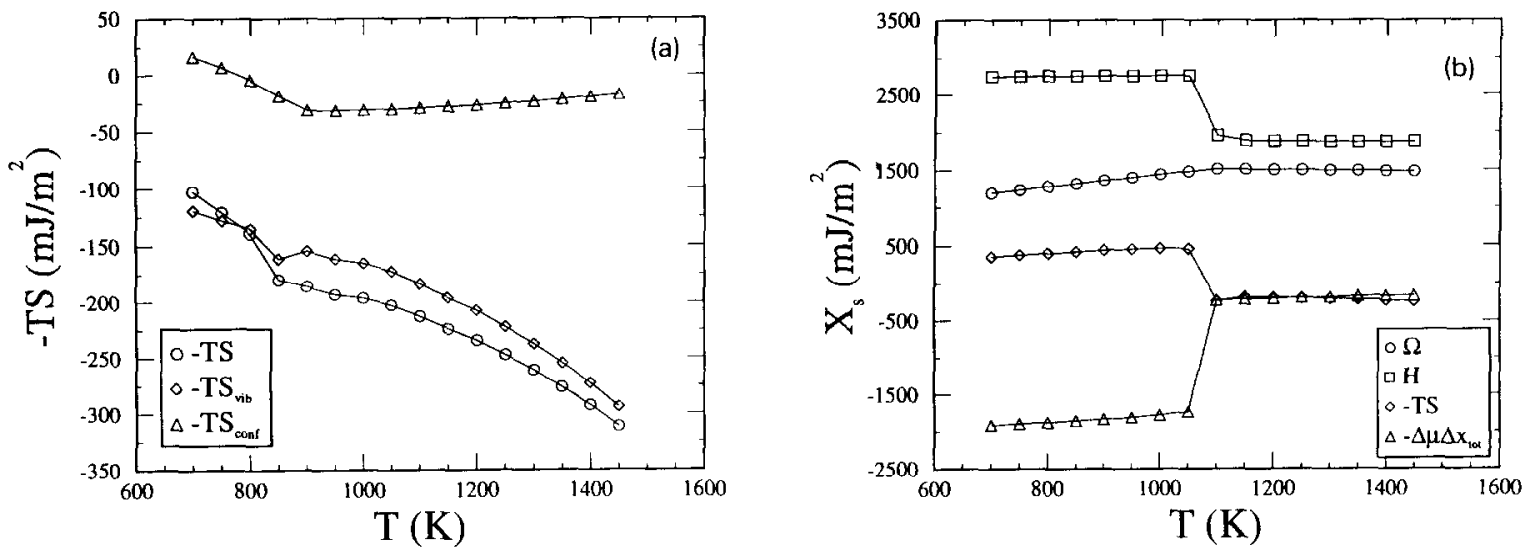

Fig. 7. Total entropic contribution to the surface free energy and its vibrational and configurational components as a function of temperature for (a) $x_{\mathrm{B}}=5 \% \mathrm{Pt}$ and (b) $x_{\mathrm{B}}=95 \% \mathrm{Pt}$. 
directions in the ordered surface become the [110] and [110] orientations in the fcc structure of the bulk, respectively.

In order to identify the driving force for the observed surface phase transitions we compare the contributions of the surface enthalpy, entropy, and segregation to the surface grand potential free energy, $\Omega_{\text {surf }}$, at temperatures immediately above and below the transition temperature. The surface entropy, $S_{\text {surf }}=$ $-\left(\partial \Omega_{\text {surf }} / \partial T\right)_{\Delta \mu}$ is calculated numerically by performing simulations at fixed $\Delta \mu$ at different temperatures. Fig. 6 shows the variation in the grand potential free energy and its different components with temperature for the $5 \%$ and $95 \% \mathrm{Pt}$ bulk compositions. At the surface order-disorder phase transition for the Ni-rich compositions, the slopes of all three components of the surface free energy (i.e., the surface enthalpy, the surface entropy, and the energy due to the platinum segregation to the surface) change discontinuously due to the second order character of the transitions (see figs. $6 a$ and $7 a$ ). The surface entropy and the energy due to the platinum segregation to the surface, $-\Delta \mu \Delta x_{\text {total }}\left(\Delta x_{\text {total }}\right.$ is the total segregation to the surface region), increase the surface free energy as the system is cooled. On the other hand, the surface enthalpy decreases the surface free energy on cooling. The rate of decrease of the surface enthalpy and the rate of increase of the surface entropy and the energy due to $\mathrm{Pt}$ segregation, all increase sharply as the system is cooled through the order-disorder transition temperature. Therefore, we conclude that the surface order-disorder phase transitions on the Ni-rich side of the phase diagram is essentially enthalpic in nature. A similar analysis of the surface free energy near the order-disorder transition on the Pt-rich side of the phase diagram (see fig. 6b), shows that both the surface enthalpy and total entropy favor a disordered surface whereas the energy due to the Ni segregation to the surface region favors surface ordering.

The surface entropies, in both the Ni-rich and Pt-rich alloys, do not favor the surface phase transitions when the temperature is lowered, as described above (see fig. 6). Since the configura- tional entropy of a disordered surface is larger than that of an ordered surface, the configurational surface entropy also does not favor the ordering of the surface transitions. In order to determine the effects of the vibrational entropy on the surface phase transitions, we examine the variation in both the vibrational and configurational entropic contributions to the surface free energy as a function of the temperature (see fig. 7). It is evident that on both sides of the phase diagram, that the surface vibrational entropy also favors disordered surfaces.

As was shown in the previous section, the (100) surface order-disorder transition temperatures for both the $\mathrm{Ni}$ - and Pt-rich alloys are well above the bulk order-disorder transitions. A previous study [8] of a stoichiomctric $\mathrm{A}_{3} \mathrm{~B}$ alloy (performed using a lattice gas model and the cluster-variation method), showed that the (100) surface may order at a temperature well above or below the bulk order-disorder transition temperature depending on the strength of the ordering tendency in the bulk. However, that study neglected the effects of atomic vibrations and surface relaxation. While their conclusion [8] that the (100) surface may order above or below the bulk transition temperatures should remain valid, inclusion of the atomic vibrations and surface relaxation may significantly change the magnitude of surface order-disorder transition temperatures.

The results presented in section 3 demonstrate that the effects of neglecting atomic vibrations in the surface segregation calculations are very serious and would lead to erroneous results. The effects of neglecting surface relaxation, on the other hand, is rather small. One of the effects of including atomic vibration in Monte Carlo or molecular dynamics simulations, or including it in an approximate form such as in this study, is the variation in lattice constant with temperature. In an attempt to determine whether the main effect of atomic vibration on the surface concentration profile can be captured by simply rescaling the lattice constant to its appropriate, finite temperature value we rescaled all of the atomic positions of the cleaved, unrelaxed crystal by the $T=1500$ $\mathrm{K}$ equilibrium lattice constant (which was deter- 
mined including atomic vibrations). The equilibrium concentrations were determined by minimizing the grand potential with respect to the atomic site concentration with the atoms fixed at these positions. The resultant $\mathrm{Pt}$ concentrations on the first through fourth (002) atomic plane at $T=$ $1500 \mathrm{~K}$ and $x_{\mathrm{B}}=10 \% \mathrm{Pt}$ were $8.50 \%, 11.07 \%$, $9.79 \%$ and $10.05 \%$, respectively. Comparison with the fully relaxed concentration profile (including atomic vibration and atomic relaxation - case D), presented in table 1 , shows that including the thermal expansion by rescaling the lattice constant does not capture the effects of atomic vibration on the segregation profile. In this case, we find that the nickel concentration on the first (002) plane increases by $1.5 \%$ and on the second plane decreases by $0.4 \%$, whilc the cquilibrium segregation determined with the full relaxations (case D) show the reverse segregation tendency: i.e., the nickel concentration on the first (002) plane is decreased by $2 \%$ and on the second plane it is increased by $0.65 \%$. Similar calculation on the (111) surface at $1500 \mathrm{~K}$ and $x_{\mathrm{B}}=10 \% \mathrm{Pt}$, where atoms were fixed at the fcc lattice positions corresponding to the bulk lattice constant at 1500 $\mathrm{K}$, shows the $\mathrm{Pt}$ concentrations on the first four planes are $7.07 \%, 11.56 \%, 9.64 \%$ and $10.14 \%$. Again, comparison with the data in table 2 (case D) further demonstrates that the effects of atomic vibrations on the surface segregation cannot be absorbed by a simple lattice constant rescaling procedure. While simply rescaling the atomic coordinates by the finite temperature lattice parameter does not yield the proper segregation profile, it does reasonably predict the overall magnitude of the segregation (compare these results with case A).

A major point that has been ignored in several of the previous surface segregation studies, is that the calculations should be carried out at temperatures and bulk compositions corresponding to points in the single phase regions rather than two phase regions of the phase diagram. The segregation profiles reported earlier [9], employing the same EAM potentials used in the present study, were determined at points in two phase regions of the EAM phase diagram. Indeed, the author of ref. [9] recognized that those simulations had the fundamental flaw that the bulk concentrations were oscillatory far below the surface.

In summary, we have investigated the orderdisorder phase transitions and segregation at the (001) surface of a series of $\mathrm{Ni}-\mathrm{Pt}$ alloys using EAM interatomic potentials and a recently introduced free energy simulation method. On the $\mathrm{Ni}$-rich side of the phase diagram, we observe second order surface order-disorder phase transitions in which the first (002) plane becomes ordered with the $\mathrm{c}(2 \times 2)$ pattern and the second plane becomes nearly pure $\mathrm{Ni}$. On the Pt-rich side of the phase diagram, we observe first order surface order-disorder phase transition in which the first and second (002) planes become nearly pure $\mathrm{Ni}$ and nearly pure $\mathrm{Pt}$, respectively. In both cases, the surface order-disorder phase transition occurs well above the bulk phase transition temperatures. The present simulations have also demonstrated the overriding importance of including atomic vibrations in analyses of surface segregation.

\section{Acknowledgments}

We gratefully acknowledge the support of the Division of Materials Science of the Office of Basic Energy Sciences of the US Department of Energy, Grant No. FG02-88ER45367.

\section{References}

[1] T.M. Buck, G.H. Wheatley and L. Marchut, Phys. Rev. Lett. 51 (1983) 43.

[2] K.S. Liang, H.H. Hung, S.L. Chang, Z. Fu, S.C. Moss and K. Oshima, Surface X-Ray and Neutron Scattering, Eds. H. Zabel and I.K. Robinson (Springer, Berlin, 1992); S.B. Rivers, W.N. Unertl, H.H. Hung and K.S. Liang, Mater. Res. Soc. Symp. Proc. 205 (1992) 145;

S.B. Rivers, W.N. Unertl, H.H. Hung and K.S. Liang, in: MRS Fall Proceedings (1990).

[3] Y. Liu and P. Wynblatt, Surf. Sci. Lett. 241 (1991) L21.

[4] J. Eugene, B. Aufray and F. Cahane, Surf. Sci. 241 (1991) 1.

[5] G. Treglia and B. Legrand, Phys. Rev. B 35 (1987) 4338.

[6] S.M. Foiles, Phys. Rev. B 12 (1985) 7983.

[7] J. Eymery and J.C. Joud, Surf. Sci. 231 (1990) 419. 
[8] J.M. Sanchez and J.L. Moran-Lopez, Phys. Rev. B 32 (1985) 3534.

[9] M. Lundberg, Phys. Rev. B 36 (1987) 4692.

[10] V. Kumar, D. Kumar and S.K. Joshi, Phys. Rev. B 19 (1979) 1954.

[11] Y. Teraoka, Surf. Sci. Lett. 238 (1990) L453.

[12] R. Najafabadi, H.Y. Wang, D.J. Srolovitz and R. LeSar, Acta Metall. Mater. 39 (1991) 3701.

[13] S.M. Foiles, M.I. Baskes and M.S. Daw, Phys. Rev. B 12 (1986) 7983.

[14] Y. Gauthier, W. Hoffmann and M. Wuttig, Surf. Sci. 233 (1990) 239.

[15] Y. Gauthier, Y. Joly, R. Baudoing and J. Rundgren, Phys. Rev. B 31 (1985) 6215.
[16] Y. Gauthier, R. Baudoing and J. Jupille, Phys. Rev. B 40 (1989) 1500.

[17] Y. Gauthier, R. Baudoing, M. Lundberg and J. Rundgren, Phys. Rev. B 35 (1987) 7867.

[18] D. Dufayard, R. Baudoing and Gauthier, Surf. Sci. 233 (1990) 223

[19] R. LeSar, R. Najafabadi and D.J. Srolovitz, Phys. Rev. Lett. 63 (1989) 624.

[20] R. Najafabadi, D.J. Srolovitz and R. LeSar, J. Mater. Res. 5 (1990) 2663.

[21] R. Najafabadi, D.J. Srolovitz and R. LeSar, J. Mater. Res. 6 (1991) 999. 